\section{Effect of daratumumab on normal plasma cells, polyclonal immunoglobulin levels, and vaccination responses in extensively pre-treated multiple myeloma patients}

The CD38-targeting antibody daratumumab has strong activity against multiple myeloma (MM), but is associated with a modest increase in infectious complications. ${ }^{1,2}$ Normal plasma cells (PC) are an important part of humoral immunity because they produce the majority of serum antibodies which contribute to the protection against a wide range of pathogens and toxins. ${ }^{3}$ The effect of daratumumab on normal PC is currently unknown. A better understanding of this is critical in order to provide recommendations on infectious prophylaxis, including vaccinations. Here, we show that daratumumab reduced the frequency of normal PC in bone marrow (BM) samples from patients with relapsed/refractory (RR) MM and that this was accompanied by reduced levels of polyclonal IgA, IgE and IgM. Polyclonal IgG levels remained unchanged, which may be explained by a subset of normal PC with reduced CD38 expression that survived during daratumumab therapy. In addition, daratumumab-

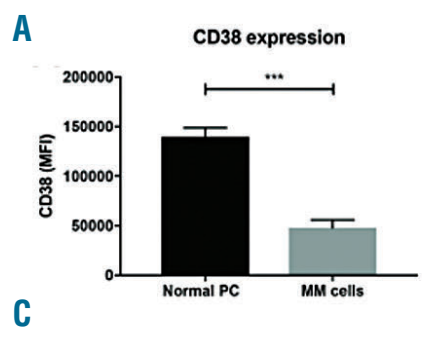

C
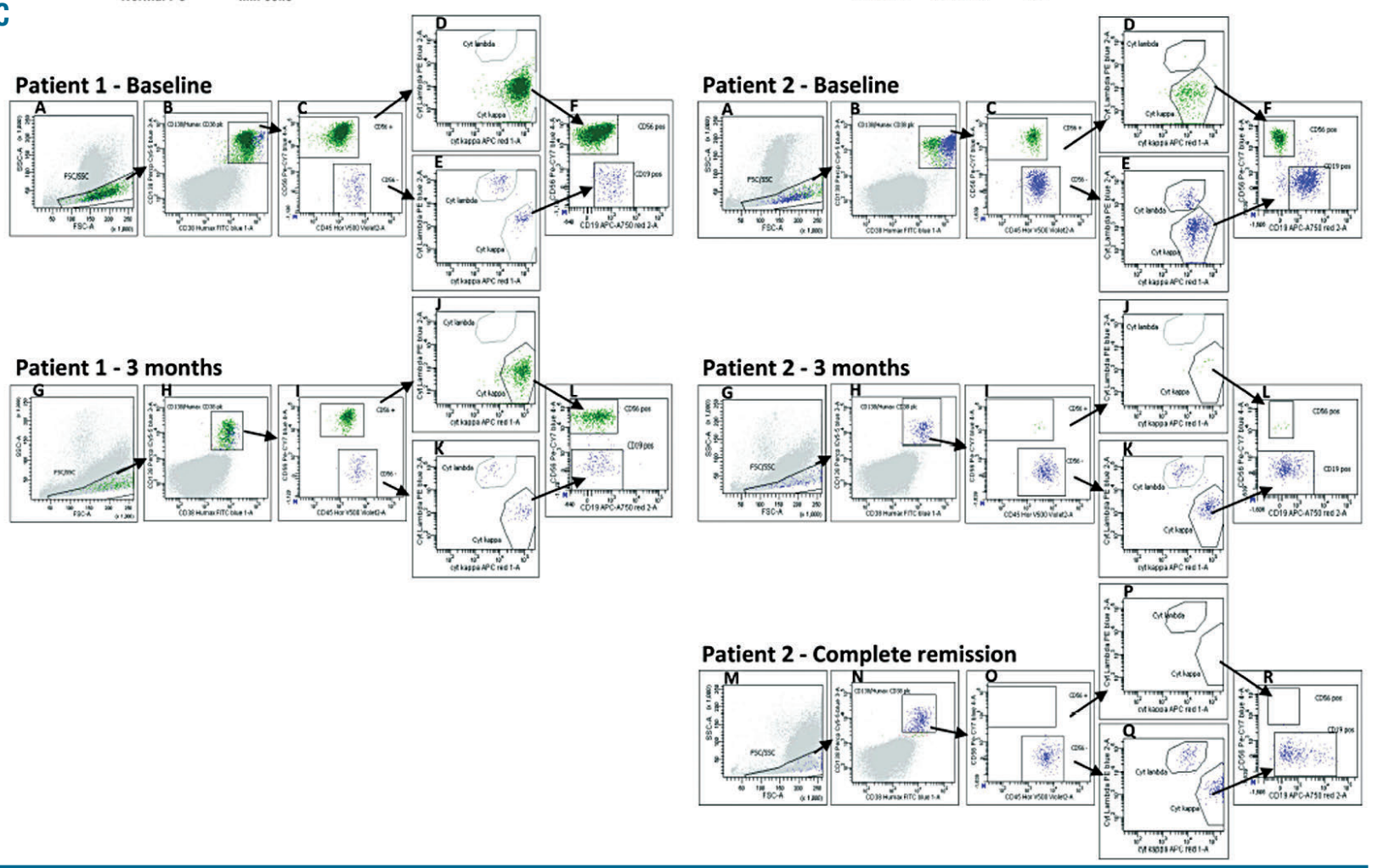

B

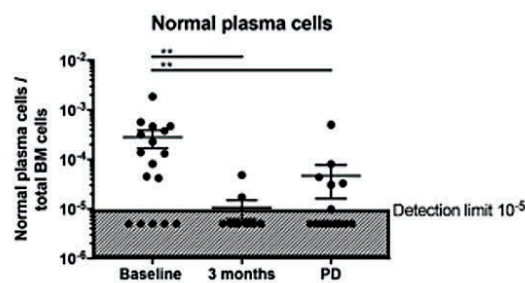

Figure 1. The effect of daratumumab treatment on normal plasma cells in patients with relapsed/refractory multiple myeloma. (A) Levels of expression of CD38 on the cell surface were analyzed by flow cytometry on normal plasma cells (PC) in bone marrow (BM) samples obtained from five healthy donors and on multiple myeloma (MM) cells in BM samples obtained from 25 patients with daratumumab-naïve relapsed/refractory (RR) MM. PC and MM cells were isolated from BM aspirates by Ficoll-Hypaque density-gradient centrifugation. Data are depicted as mean \pm standard error mean (SEM). The difference between the indicated groups was calculated using the Mann-Whitney U-test, $* * * P<0.001$. (B) The frequency of normal PC in sequential BM samples obtained from 17 RRMM patients: prior to initiation of daratumumab monotherapy, after 3 months of daratumumab treatment, and at the time of disease progression. Normal $\mathrm{PC}$ and MM cells were detected with a minimum sensitivity of 1 in $10^{5}$ nucleated cells. Dots represent individual data, lines indicate mean $\pm \mathrm{SEM}$. Levels below the lowest level of detection (LLOD) were set at the LLOD for statistical analysis, and at 50\% of the LLOD for purposes of visualization. Differences between the indicated groups were calculated using the Wilcoxon matched-pairs signed ranks test; $* * P<0.01$. (C) Two representative examples show the gating strategy used for the identification of normal PC and MM cells, in BM samples obtained before the initiation of daratumumab treatment and after 3 months of treatment. In patient 2, BM was also analyzed to confirm complete response. PC were identified based on CD38 and CD138 surface expression after preselection in a forward and side scatter plot. Malignant PC were distinguished from normal PC based on CD56, CD19 and CD45 expression. Malignant PC (green) were monoclonal for $\mathrm{k}$ light chains in both patients, while normal PC (blue) showed polyclonality based on $\mathrm{k} / \lambda$ staining. The dot plots also show that normal, CD $19^{+} \mathrm{PC}$ had reduced surface expression of CD38 and CD19 following daratumumab treatment (CD38: compare plots $\mathrm{H}$ and N with B; CD19: compare plots L and R with F). CD38 expression was assessed using an antibody that binds to an epitope on CD38 distinct from the one bound by daratumumab (Humax-003 FITC, Janssen Pharmaceuticals). 
treated RRMM patients produced protective IgG antibody titers following vaccination against Streptococcus (S.) pneumoniae, Haemophilus (H.) influenzae type B and seasonal influenza, to a similar extent as observed in daratumumab-naïve RRMM patients.

We first examined the levels of expression of CD38 on the surface of normal PC in BM samples from healthy controls, and on MM cells in BM samples obtained from RRMM patients, treated in the GEN501 study, before ini- tiation of daratumumab monotherapy (NCT00574288) (Online Supplementary Table S1). ${ }^{4}$ The CD38 expression on normal PC from healthy donors $[\mathrm{n}=5$; median fluorescence intensity (MFI) 141,288; range 112,426-164,626)] was significantly higher than that on MM cells from daratumumab-naïve RRMM patients ( $n=25$; MFI 36,174; range 6,997-160,005; $P=0.0002$ ) (Figure 1A). We therefore hypothesized that normal PC are susceptible to daratumumab-mediated lysis, and assessed the frequency of
A

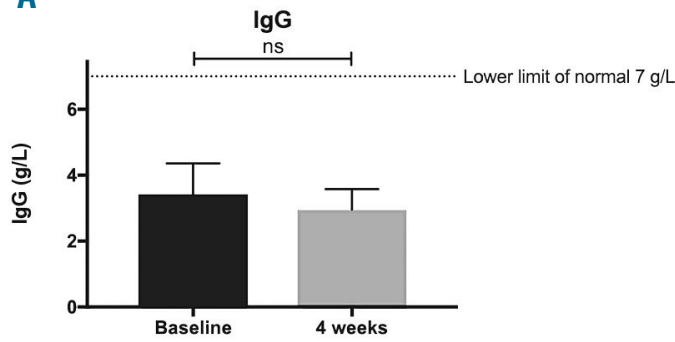

C

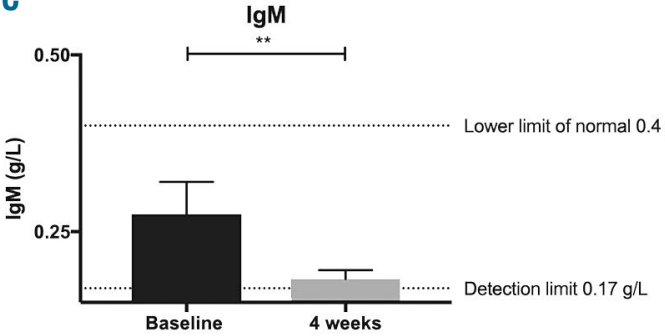

E

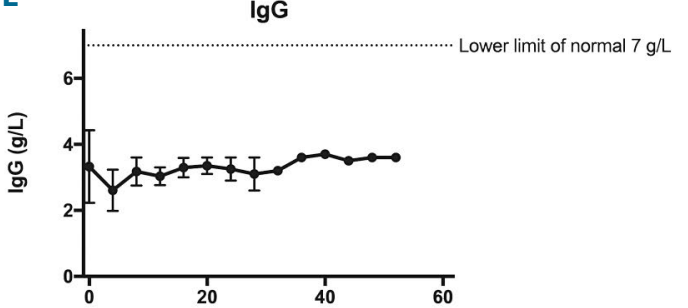

G

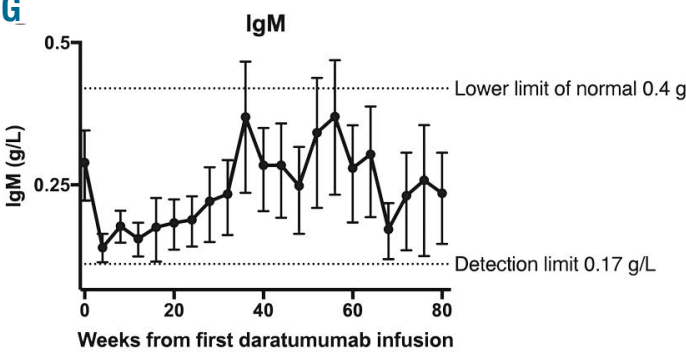

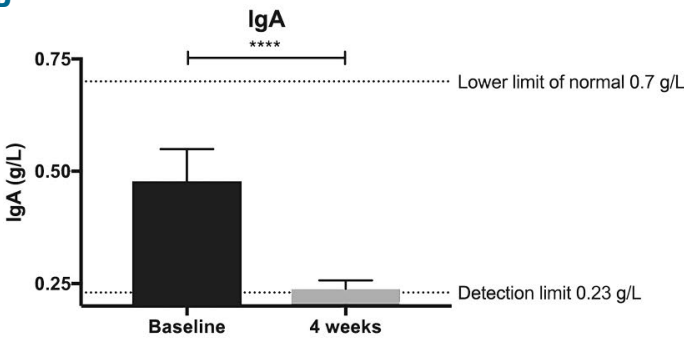

D

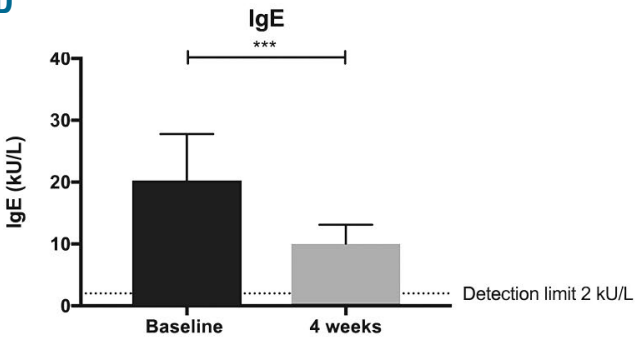

$\mathrm{F}$

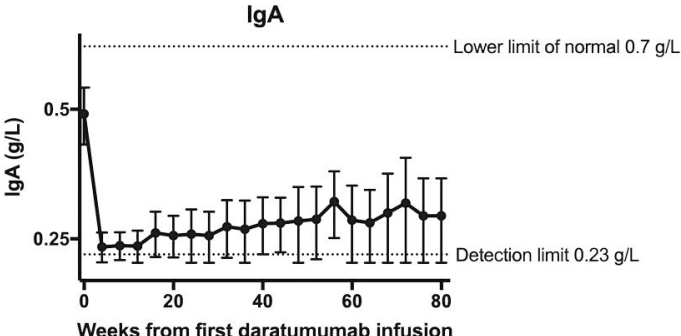

$\mathrm{H}$

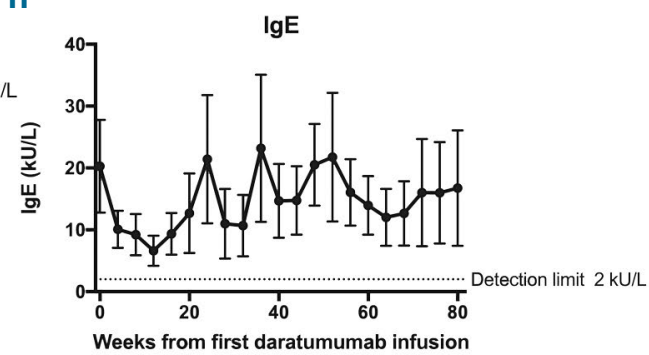

Figure 2. The effect of daratumumab treatment on polyclonal immunoglobulin levels in relapsed/refractory multiple myeloma. (A-D) Serum levels of uninvolved, polyclonal IgG (A), IgA (B), IgM (C) and IgE (D) were analyzed using nephelometry, at baseline and after 4 weeks of daratumumab monotherapy in 30 patients with relapsed/refractory multiple myeloma (RRMM). Serum samples were obtained directly before the initiation of the daratumumab infusion. Data represent the mean \pm standard error of mean (SEM). $P$-values were calculated using the Wilcoxon matched ranks test; $* * P<0.01, * * * P<0.001 ; * * * * P<0.0001$. $(\mathrm{E}-\mathrm{H})$ Serum levels of uninvolved, polyclonal IgG $(\mathrm{E})$, IgA $(\mathrm{F})$, IgM $(\mathrm{G})$ and IgE $(\mathrm{H})$ were analyzed using nephelometry, at baseline and every 4 weeks during treatment with daratumumab as a single agent, in 30 RRMM patients. Serum samples were obtained directly prior to the daratumumab infusions. Follow-up was discontinued when daratumumab treatment was stopped due to disease progression. Data represent the mean \pm SEM. Patients with IgG or IgA M-protein were excluded from polyclonal IgG or IgA assessments, respectively. There were no patients with IgE or IgM M-protein. In addition, patients receiving IgG replacement therapy $(n=3$ ) were excluded from the polyclonal lgG analysis, and patients were excluded if baseline values were missing ( $n=5 \mathrm{for}$ IgE evaluation). IgA, IgM, and IgE levels below the lower limit of detection (LLOD) were set at the LLOD (IgA $0.22 \mathrm{~g} / \mathrm{L}$; IgM $0.16 \mathrm{~g} / \mathrm{L}$ and IgE $2.0 \mathrm{kU} / \mathrm{L}$ ) for statistical analysis. IgG, immunoglobulin G; IgA, immunoglobulin A; IgM, immunoglobulin M; IgE, immunoglobulin E; ns, not significant. 
A

\section{Daratumumab treated RRMM}

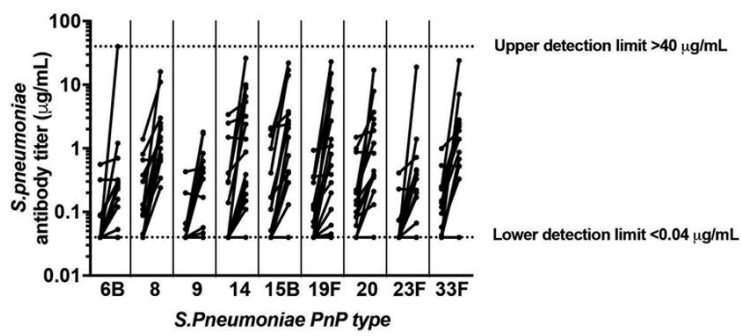

C

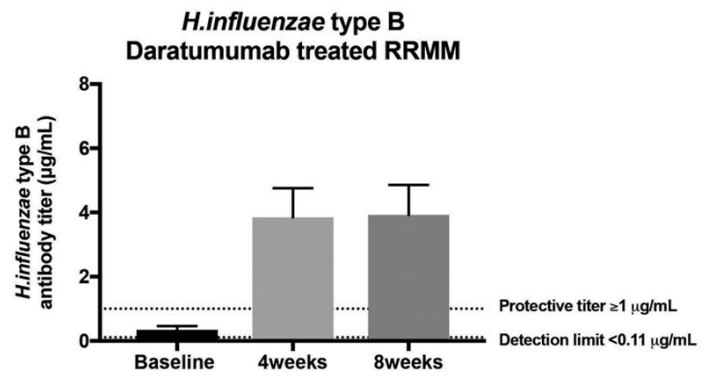

E

Influenza

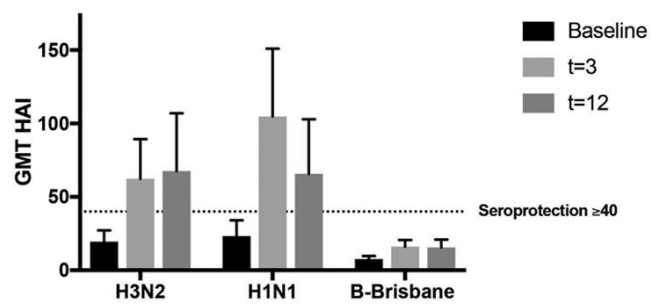

B

S.pneumoniae

Daratumumab naive RRMM

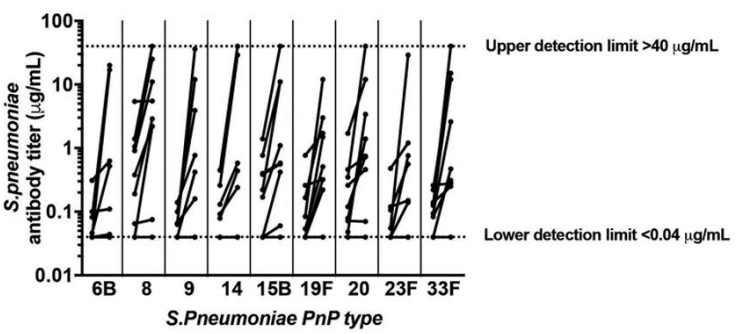

D

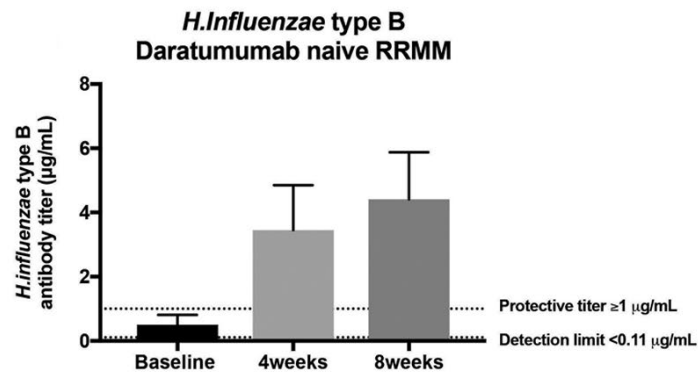

F

\begin{tabular}{|c|c|c|}
\hline & \multicolumn{2}{|c|}{$\begin{array}{c}\text { RRMM } \\
\text { Daratumumab-treated } \\
n=13\end{array}$} \\
\hline & $S P r, n=13$ & $\mathrm{SCr}, \mathrm{n}=12^{*}$ \\
\hline H3N2, n (\%) & $4(31)$ & $2(17)$ \\
\hline H1N1, n (\%) & $5(38)$ & $3(25)$ \\
\hline B-Brisbane, $n(\%)$ & $3(23)$ & $2(17)$ \\
\hline$\geq 2$ strains, $n(\%)$ & $4^{\#}(31)$ & $2(17)$ \\
\hline - $\quad H 1 N 1 \&$ H3N2 & 1 & 0 \\
\hline - $\quad$ H1N1 \& B-Brisbane & 1 & 1 \\
\hline - $\quad$ H3N2 \& B-Brisbane & 1 & 1 \\
\hline - $\quad$ All 3 & 1 & 0 \\
\hline
\end{tabular}

Figure 3. Response to S. pneumoniae, $H$. influenzae type B, and seasonal influenza vaccination in daratumumab-treated and daratumumab-naïve patients with relapsed/refractory multiple myeloma. (A) Specific lgG titers ( $\mu \mathrm{g} / \mathrm{mL})$ against pneumococcal serotypes $6 \mathrm{~B}, 8,9,14,15 \mathrm{~B}, 19 \mathrm{~F}, 20,23 \mathrm{~F}$ and 33F, assessed by enzyme-linked immunosorbent assay (ELISA), at baseline and at best response following PCV-13 and PPV-23 vaccination in 16 daratumumab-treated patients with refractory/relapsed multiple myeloma (RRMM). Connected dots represent individual patients. One patient was lost to follow-up and excluded from response evaluation. (B) Specific IgG titers $(\mu \mathrm{g} / \mathrm{mL}$ ) to the aforementioned pneumococcal serotypes, assessed by ELISA, at baseline and at best response in nine daratumumab-naïve RRMM patients. Connected dots represent individual patients. One patient was not evaluable for response due to disease progression requiring a new treatment regimen, which included daratumumab. (C) Specific lgG titers ( $\mu \mathrm{g} / \mathrm{mL}$ ) at baseline, as well as 4 and 8 weeks following $H$. influenzae type B vaccination in 17 daratumumab-treated RRMM patients. Bars represent mean titer \pm standard error of mean (SEM). (D) Specific IgG titers ( $\mu g / \mathrm{mL}$ ) at baseline, as well as 4 and 8 weeks following H.influenzae type B vaccination in ten daratumumab-naïve RRMM patients. Bars represent mean titer \pm SEM. (E) Strain-specific hemagglutinin inhibition assay geometric mean titers at baseline, as well as 3 and 12 weeks following seasonal influenza vaccination in 13 daratumumab-treated RRMM patients. Bars represent mean \pm SEM. (F) Seroprotection and seroconversion rates to the three influenza strains included in the seasonal influenza vaccination in 13 daratumumab-treated RRMM patients. *In one patient, seroconversion rates could not be assessed because of missing baseline titers. This patient had seroprotective titers against H3N2 and H1N1. \#One patient had seroprotective titers against all three strains. S. pneumoniae: Streptococcus pneumoniae; H. influenza: Haemophilus influenzae; IgG: immunoglobulin G; GMT: geometric mean titer; HAl: hemagglutinin inhibition assay; n, number of patients; $\mathrm{SPr}$, seroprotection rate, defined as the percentage of patients achieving an HAI titer $\geq 1: 40$; SCr, seroconversion rate, defined as the percentage of patients achieving $\mathrm{a} \geq 4$-fold increase in $\mathrm{HAl}$ titer.

normal PC in sequential BM samples from 17 of these 25 RRMM patients. Samples were obtained at baseline, after 3 months of daratumumab treatment, and upon disease progression. In these sequential samples, we observed a significant reduction in frequency of normal PC after 3 months of daratumumab treatment and at the time of progressive disease, when compared to baseline samples $(P=0.0078$ and $P=0.002$, respectively) (Figure $1 \mathrm{~B})$. Interestingly, normal $P C$ that survived daratumumab treatment had reduced surface expression of CD38 and CD19 (Figure 1C). This reduced CD38 expression, which was assessed using an antibody that binds to an epitope on CD38 distinct from the one bound by daratumumab, ${ }^{5}$ can partly be explained by selection of normal PC with lower CD38 levels, whereas cells with higher CD38 expression are eliminated. In addition, we have previously shown that daratumumab reduces CD38 expression via active transfer of daratumumab-CD38 complexes and 
accompanying cell membrane from target cells to monocytes and granulocytes (trogocytosis). This process may also explain the reduced CD19 expression, given its close physical proximity to CD38 on the cell surface. ${ }^{5}$ Collectively, these data indicate that daratumumab reduces the frequency of normal PC, but that some of these normal PC survive daratumumab treatment.

Next, we assessed the effect of daratumumab treatment on serum polyclonal immunoglobulin levels in sequential peripheral blood serum samples, obtained from 30 RRMM patients, who were treated with daratumumab monotherapy in the first part of the DARA/ATRA study (NCT02751255) (Online Supplementary Table S2). Uninvolved, polyclonal immunoglobulins (IgA, IgE and IgM for IgG myeloma; $\operatorname{IgG}, \operatorname{IgE}$ and $\operatorname{Ig} M$ for $\operatorname{IgA}$ myeloma; and $\operatorname{IgG}, \operatorname{IgA}, \operatorname{IgE}$ and IgM for light-chain only myeloma) were assessed using nephelometry, at baseline and every 4 weeks during daratumumab treatment until disease progression. Serum samples were obtained directly prior to the daratumum$\mathrm{ab}$ infusion. As expected in these heavily pretreated RRMM patients, polyclonal immunoglobulins were suppressed at baseline. Already after 4 weeks of daratumum$\mathrm{ab}$ treatment, we observed a significant decrease in levels of polyclonal IgA, IgM and IgE, while levels of IgG remained stable (Figure 2A-D). This reduction occurred in all patients, irrespective of the response to daratumumab treatment (data not shown). Since daratumumab is an IgG- $\kappa$ antibody with a maximum serum trough concentration of $0.5 \mathrm{~g} / \mathrm{L}$ at the end of the weekly infusions, ${ }^{6}$ there might be a small overestimation of polyclonal IgG levels. Even when corrected for this, polyclonal IgG levels at baseline and after 4 weeks remained comparable (Online Supplementary Figure S1). In addition, although the numbers of patients are limited, longitudinal analysis throughout daratumumab treatment showed that IgG levels remained stable, but below the lower limit of normal (Figure 2E). Interestingly, after the initial reduction, the levels of polyclonal IgE and IgM returned to baseline by approximately 24 and 36 weeks of daratumumab treatment, respectively. Polyclonal IgA levels did not show substantial recovery (Figure 2F-H). Our data suggest that IgG-secreting normal PC are less sensitive to daratumumab, compared to non-IgG-secreting PC. PC that express these different heavy chains have a different tissue localization: IgG-secreting PC are mainly present in the BM, while mucosa- and lymphoid tissue-associated PC also produce IgM, IgA and IgE. ${ }^{3}$ Different interactions between PC and their microenvironment, which contribute to PC survival, may explain the variability to maintain these PC subsets.

To evaluate the effect of daratumumab on antigen-specific antibody responses, we vaccinated 17 RRMM patients against $S$. pneumoniae and $H$. influenzae type B during daratumumab monotherapy (first part of DARA/ATRA study) (Online Supplementary Table S3). The median duration of daratumumab treatment at vaccination was 2 months (range 0.5-17 months). All patients received dexamethasone as prophylaxis against infusionrelated reactions. As a control group, we vaccinated ten daratumumab-naïve RRMM patients. Most patients $(80 \%)$ were treated with an immunomodulatory drugbased regimen (median duration of treatment with current regimen for these patients: 11 months (range 0-57) (Online Supplementary Table S3). All regimens contained low-dose dexamethasone, except for one, for a patient who received lenalidomide maintenance without glucocorticoids. S. pneumoniae vaccination consisted of the conjugated PCV-13 vaccine (Prevenar, Pfizer) followed by the polysaccharide PPV-23 vaccine (Pneumovax, Merck Sharp \& Dohme), both administered intramuscularly with an 8-week interval. ${ }^{7}$ Specific antibody titers were measured using an enzyme-linked immunoabsorbent assay (ELISA) at baseline, as well as 4 and 8 weeks after each vaccine. Response was defined as an absolute titer $\geq 2 \mu \mathrm{g} / \mathrm{mL}$ or $\mathrm{a} \geq 2$-fold increase in six out of nine analyzed pneumococcal subtypes $(6 \mathrm{~B}, 8,9,14,15 \mathrm{~B}, 19 \mathrm{~F}, 20,23 \mathrm{~F}$ and $33 \mathrm{~F}){ }^{8} \mathrm{H}$. influenzae vaccination consisted of a single intramuscular dose of Act-Hib (Sanofi), and specific antibody titers were assessed using ELISA at baseline, as well as 4 and 8 weeks following vaccination. Response was defined as an absolute titer $\geq 1 \mu \mathrm{g} / \mathrm{mL}$, or a $\geq 4$-fold increase in titer (for details see the Online Supplementary Methods). ${ }^{8}$ No differences in response rates following $S$. pneumoniae and $H$. influenzae type $\mathrm{B}$ vaccinations were observed between daratumumab-treated $(68.8 \%$ and $66.7 \%$, respectively) and daratumumab-naïve patients $(55.6 \%$ and $62.5 \%$, respectively; $P=0.509$ and $P=0.842)$ (Figure $3 \mathrm{~A}-\mathrm{D}$ ). In addition, three patients (2 daratumumab-treated and 1 daratumumab-naïve patient) had protective titers against $H$. influenzae at baseline, and retained immunity. ${ }^{8}$ These results are similar to, or better than, those previously reported in less heavily pretreated $\mathrm{MM}$ patients. ${ }^{8-11}$

In addition, 13 of the 17 daratumumab-treated patients received trivalent seasonal influenza vaccination during seasons 2016/2017 and 2017/2018. Serum samples were collected at baseline, as well as 3 and 12 weeks following vaccination. Hemagglutinin inhibition assays were performed according to standard procedures (Online Supplementary Methods). ${ }^{12}$ Seroprotection was defined as an absolute titer $\geq 1: 40$, and seroconversion as $a \geq 4$-fold increase in titer. ${ }^{12}$ Seroprotection rates were $31 \%$ for $\mathrm{H} 3 \mathrm{~N} 2,38 \%$ for $\mathrm{H} 1 \mathrm{~N} 1$ and $23 \%$ for B-Brisbane. In addition, four patients $(31 \%)$ developed seroprotective titers against two or more strains, of whom one patient was protected against all three strains (Figure 3E, F). Seroconversion rates were $17 \%$ for $\mathrm{H} 3 \mathrm{~N} 2,25 \%$ for $\mathrm{H} 1 \mathrm{~N} 1$ and $17 \%$ for B-Brisbane. Two patients showed seroconversion for $\geq 2$ strains (Figure $3 \mathrm{~F}$ ). Although we did not perform serological analyses in daratumumabnaïve RRMM patients, prior studies have found similar response rates. Further research is needed to improve the efficacy of this vaccine for MM patients. ${ }^{11,13,14}$

During follow-up (median 17 months; range 6-21), 14 out of 17 daratumumab-treated patients and six out of ten daratumumab-naïve patients developed infectious complications. Importantly, only one daratumumabtreated patient developed pneumonia caused by $H$. influenzae type $\mathrm{B}$. This patient did not develop protective antibody titers against $H$. influenzae following vaccination. The infection resolved completely with antibiotic treatment. To the best of our knowledge, none of the other infections was caused by $S$. pneumoniae or influenza (for details, see the Online Supplementary Methods).

In conclusion, our data show that daratumumab reduces the frequency of normal PC, which is reflected in reduced levels of polyclonal IgA, IgE and IgM upon initiation of daratumumab treatment. However, our data also demonstrate that a proportion of normal PC persists during daratumumab treatment, which may be related to downregulation of CD38 on their cell surface. These resistant, normal PC are likely responsible for the stable levels of polyclonal IgG during treatment. The recovery of polyclonal $\operatorname{IgM}$ and $\operatorname{IgE}$ to baseline levels, as well as the generation of protective $\operatorname{IgG}$ antibodies following vaccination during daratumumab therapy, to a similar extent as observed in daratumumab-naïve patients, indi- 
cates that B cells can differentiate into PC during daratumumab treatment, which is in contrast to data obtained from vaccination studies with B-cell-depleting therapies such as rituximab. ${ }^{15}$ We therefore recommend routine vaccination, also in patients undergoing daratumumab treatment, to reduce infection-related morbidity and mortality. Furthermore, daratumumab-treated patients with low IgG levels and recurrent infections may benefit from IgG replacement therapy.

Kristine A. Frerichs, ${ }^{1}$ Patricia W.C. Bosman, Jeroen F. van Velzen, ${ }^{2}$ Pieter L.A. Fraaij, ${ }^{3}$ Marion P.G. Koopmans, ${ }^{3}$ Guus F. Rimmelzwaan, ${ }^{3}$ Inger S. Nijhof, Andries C. Bloem, ${ }^{2}$ Tuna Mutis, ${ }^{1}$ Sonja Zweegman ${ }^{1}$ and Niels W.C.J. van de Donk

${ }^{1}$ Amsterdam UMC, Vrije Universiteit Amsterdam, Department of Hematology, Cancer Center Amsterdam, Amsterdam; ${ }^{2}$ University Medical Center, Laboratory of Translational Immunology, Utrecht and ${ }^{3}$ Erasmus Medical Center, Department of Viroscience, National Influenza Center, Rotterdam, the Netherlands.

${ }^{\circ}$ Current address: University of Veterinary Medicine, Center for Emerging Infections and Zoonoses, Hannover, Germany

Funding: the DARA/ATRA study was supported by research funding from Janssen Pharmaceuticals.

Correspondence: Niels W.C.J. van de Donk

n.vandedonk@amsterdamumc.nl

doi:10.3324/haematol.2019.231860

Information on authorship, contributions, and financial \& other disclosures was provided by the authors and is available with the online version of this article at www. haematologica.org.

\section{References}

1. Dimopoulos MA, San-Miguel J, Belch A, et al. Daratumumab plus lenalidomide and dexamethasone versus lenalidomide and dexamethasone in relapsed or refractory multiple myeloma: updated analysis of POLLUX. Haematologica. 2018;103(12):2088-2096.

2. Moreau P, Attal M, Hulin C, et al. Bortezomib, thalidomide, and dexamethasone with or without daratumumab before and after autologous stem-cell transplantation for newly diagnosed multiple myeloma (CASSIOPEIA): a randomised, open-label, phase 3 study. Lancet. 2019;394(10192):29-38

3. Radbruch A, Muehlinghaus G, Luger EO, et al. Competence and competition: the challenge of becoming a long-lived plasma cell. Nat
Rev Immunol. 2006;6(10):741-750.

4. Lokhorst HM, Plesner T, Laubach JP, et al. Targeting CD38 with daratumumab monotherapy in multiple myeloma. N Engl J Med. 2015;373(13):1207-1219.

5. Krejcik J, Frerichs KA, Nijhof IS, et al. Monocytes and granulocytes reduce CD38 expression levels on myeloma cells in patients treated with daratumumab. Clin Cancer Res. 2017;23(24):7498-7511.

6. Clemens PL, Yan X, Lokhorst HM, et al. Pharmacokinetics of daratumumab following intravenous infusion in relapsed or refractory multiple myeloma after prior proteasome inhibitor and immunomodulatory drug treatment. Clin Pharmacokinet. 2017;56(8):915-924.

7. CDC. Use of 13-valent pneumococcal conjugate vaccine and 23valent pneumococcal polysaccharide vaccine for adults with immunocompromising conditions: recommendations of the Advisory Committee on Immunization Practices (ACIP). MMWR 2012 12-10-2012 [cited 2019; Available from https://www.cdc.gov/mmwr/preview/mmwrhtml/mm6140a4.htm

8. Palazzo M, Shah GL, Copelan O, et al. Revaccination after autologous hematopoietic stem cell transplantation is safe and effective in patients with multiple myeloma receiving lenalidomide maintenance. Biol Blood Marrow Transplant. 2018;24(4):871-876.

9. Hinge M, Ingels HA, Slotved HC, Molle I. Serologic response to a 23valent pneumococcal vaccine administered prior to autologous stem cell transplantation in patients with multiple myeloma. APMIS 2012;120(11):935-940.

10. Karlsson J, Hogevik H, Andersson K, Roshani L, Andréasson B, Wennerås C. Pneumococcal vaccine responses in elderly patients with multiple myeloma, Waldenstrom's macroglobulinemia, and monoclonal gammopathy of undetermined significance. Trials Vaccinol. 2013;2(Suppl C):31-38.

11. Robertson JD, Nagesh K, Jowitt SN, et al. Immunogenicity of vaccination against influenza, Streptococcus pneumoniae and Haemophilus influenzae type B in patients with multiple myeloma. Br J Cancer. 2000;82(7):1261-1265.

12. Wumkes ML, van der Velden AM, Los M, et al. Serum antibody response to influenza virus vaccination during chemotherapy treatment in adult patients with solid tumours. Vaccine. 2013;31(52):6177-6184.

13. Hahn M, Schnitzler P, Schweiger B, et al. Efficacy of single versus boost vaccination against influenza virus in patients with multiple myeloma. Haematologica. 2015;100(7):e285-288.

14. Branagan AR, Duffy E, Albrecht RA, et al. Clinical and serologic responses after a two-dose series of high-dose influenza vaccine in plasma cell disorders: a prospective, single-arm trial. Clin Lymphoma Myeloma Leuk. 2017;17(5):296-304.

15. van Aalst M, Langedijk AC, Spijker R, de Bree GJ, Grobusch MP, Goorhuis A. The effect of immunosuppressive agents on immunogenicity of pneumococcal vaccination: a systematic review and meta-analysis. Vaccine. 2018;36(39):5832-5845. 